\title{
A lower bound for the Laplacian eigenvalues of a graph - proof of a conjecture by Guo
}

\author{
A. E. Brouwer \& W. H. Haemers
}

2008-02-28

\begin{abstract}
We show that if $\mu_{j}$ is the $j$-th largest Laplacian eigenvalue, and $d_{j}$ is the $j$-th largest degree $(1 \leq j \leq n)$ of a connected graph $\Gamma$ on $n$ vertices, then $\mu_{j} \geq d_{j}-j+2(1 \leq j \leq n-1)$. This settles a conjecture due to Guo.
\end{abstract}

\section{Introduction}

Let $\Gamma$ be a finite simple (undirected, without loops) graph on $n$ vertices. Let $X=V \Gamma$ be the vertex set of $\Gamma$. Write $x \sim y$ to denote that the vertices $x$ and $y$ are adjacent. Let $d_{x}$ be the degree (number of neighbors) of $x$.

The adjacency matrix $A$ of $\Gamma$ is the $0-1$ matrix indexed by $X$ with $A_{x y}=1$ when $x \sim y$ and $A_{x y}=0$ otherwise. The Laplacian matrix of $\Gamma$ is $L=D-A$, where $D$ is the diagonal matrix given by $D_{x x}=d_{x}$, so that $L$ has zero row and column sums.

The eigenvalues of $A$ are called eigenvalues of $\Gamma$. The eigenvalues of $L$ are called Laplacian eigenvalues of $\Gamma$. Since $A$ and $L$ are symmetric, these eigenvalues are real. Since $L$ is positive semidefinite (indeed, for any vector $u$ indexed by $X$ one has $u^{\top} L u=\sum\left(u_{x}-u_{y}\right)^{2}$ where the sum is over all edges $x y$ ), it follows that the Laplacian eigenvalues are nonnegative. Since $L$ has zero row sums, 0 is a Laplacian eigenvalue. In fact the multiplicity of 0 as eigenvalue of $L$ equals the number of connected components of $\Gamma$.

Let $\mu_{1} \geq \mu_{2} \geq \ldots \geq \mu_{n}=0$ be the Laplacian eigenvalues. Let $d_{1} \geq d_{2} \geq \ldots \geq d_{n}$ be the degrees, ordered nonincreasingly. We will prove that $\mu_{i} \geq d_{i}-i+2$ with basically one exception.

\section{Exception}

Suppose $\mu_{m}=0<d_{m}-m+2$. Then $d_{m} \geq m-1$, and we find a connected component with at least $m$ vertices, hence with at least $m-1$ nonzero Laplacian eigenvalues. It follows that this component has size precisely $m$, and hence $d_{1}=\ldots=d_{m}=m-1$, and the component is $K_{m}$. Now $\Gamma=K_{m}+(n-m) K_{1}$ is the disjoint union of a complete graph on $m$ vertices and $n-m$ isolated points. We'll see that this is the only exception. 


\section{Interlacing}

Suppose $M$ and $N$ are real symmetric matrices of order $m$ and $n$ with eigenvalues $\lambda_{1}(M) \geq \ldots \geq \lambda_{m}(M)$ and $\lambda_{1}(N) \geq \ldots \geq \lambda_{n}(N)$, respectively. If $M$ is a principal submatrix of $N$, then it is well known that the eigenvalues of $M$ interlace those of $N$, that is,

$$
\lambda_{i}(N) \geq \lambda_{i}(M) \geq \lambda_{n-m+i}(N) \text { for } i=1, \ldots, m .
$$

Less well-known, (see for example [3]) is that these inequalities also hold if $M$ is the quotient matrix of $N$ with respect to some partition $X_{1}, \ldots, X_{m}$ of $\{1, \ldots, n\}$. This means that $\left(M_{i, j}\right)$ equals the average row sum of the block of $N$ with rows indexed by $X_{i}$ and columns indexed by $X_{j}$.

Let $K$ be the point-line incidence matrix of a graph $\Gamma$. Then the Laplacian of $\Gamma$ is $L=K K^{\top}$. But $K K^{\top}$ has the same nonzero eigenvalues as $K^{\top} K$, and interlacing for that latter matrix implies that the eigenvalues of $L$ do not increase when an edge of $\Gamma$ is deleted.

\section{The lower bound}

Theorem 1 Let $\Gamma$ be a finite simple graph on $n$ vertices, with vertex degrees $d_{1} \geq d_{2} \geq \ldots \geq d_{n}$, and Laplacian eigenvalues $\mu_{1} \geq \mu_{2} \geq \ldots \geq$ $\mu_{n}$. If $\Gamma$ is not $K_{m}+(n-m) K_{1}$, then $\mu_{m} \geq d_{m}-m+2$.

For the union of $K_{m}$ and some isolated points we have $\mu_{m}=0$ and $d_{m}=m-1$.

The case $m=1$ of this theorem ( $\mu_{1} \geq d_{1}+1$ if there is an edge) is due to Grone \& Merris [1]. The case $m=2\left(\mu_{2} \geq d_{2}\right.$ if the number of edges is not 1) is due to Li \& Pan [4]. The case $m=3$ is due to Guo [2], and he also conjectured the general result.

Let us separate out part of the proof as a lemma.

Lemma 2 Let $S$ be a set of vertices in the graph $\Gamma$ such that each vertex in $S$ has at least e neighbors outside $S$. Let $m=|S|, m>0$. Then $\mu_{m} \geq e$. If $S$ contains a vertex adjacent to all other vertices of $S$, and $e>0$, then $\mu_{m} \geq e+1$.

Proof Consider the principal submatrix $L_{S}$ of $L$ with rows and columns indexed by $S$. Let $L(S)$ be the Laplacian of the subgraph induced on $S$. Then $L_{S}=L(S)+D$ where $D$ is the diagonal matrix such that $D_{s s}$ is the number of neighbors of $s$ outside $S$. Since $L(S)$ is positive semidefinite and $D \geq e I$, all eigenvalues of $L_{S}$ are not smaller than $e$, and by interlacing $\mu_{m} \geq e$.

Now suppose that $S=\left\{s_{0}\right\} \cup T$, where $s_{0}$ is adjacent to all vertices of $T$. Throw away all edges entirely outside $S$, and possibly also some edges leaving $S$, so that each vertex of $S$ has precisely $e$ neighbours outside $S$. Also throw away all vertices outside $S$ that now are isolated. Since these operations do not increase $\mu_{m}$, it suffices to prove the claim for the resulting situation. 
Consider the quotient matrix $Q$ of $L$ for the partition of the vertex set $X$ into the $m+1$ parts $\{s\}$ for $s \in S$ and $X \backslash S$. We find, with $r=|X \backslash S|$,

$$
Q=\left(\begin{array}{ccc}
e+m-1 & -1 \ldots-1 & -e \\
-1 & & -e \\
\vdots & L_{T} & \vdots \\
-1 & & -e \\
-e / r & -e / r \ldots-e / r & e m / r
\end{array}\right)
$$

Consider the quotient matrix $R$ of $L$ for the partition of the vertex set $X$ into the 3 parts $\left\{s_{0}\right\}, T, X \backslash S$. Then

$$
R=\left(\begin{array}{ccc}
e+m-1 & 1-m & -e \\
-1 & e+1 & -e \\
-e / r & -e(m-1) / r & e m / r
\end{array}\right)
$$

The eigenvalues of $R$ are $0, e+m$, and $e+m e / r$, and these three numbers are also the eigenvalues of $Q$ for (right) eigenvectors that are constant on the three sets $\left\{s_{0}\right\}, T, X \backslash S$. The remaining eigenvalues $\theta$ of $Q$ belong to (left) eigenvectors perpendicular to these, so of the form $\left(0, u^{\top}, 0\right)$ with $\sum u=0$. Now $L_{T} u=\theta u$, but $L_{T}=L(T)+(e+1) I$ and $L(T)$ is positive semidefinite, so $\theta \geq e+1$.

Since $m e / r \geq 1$ (each vertex in $S$ has $e$ neighbors outside $S$ and $|S|=m$, so at most $m e$ vertices in $X \backslash S$ have a neighbor in $S$ ), it follows that all eigenvalues of $Q$ except for the smallest are not less than $e+1$. By interlacing, $\mu_{m} \geq e+1$.

Proof (of the theorem). Since $\mu_{m} \geq 0$ we are done if $d_{m} \leq m-2$. So, suppose that $d_{m} \geq m-1$.

Let $\Gamma$ have vertex set $X$, and let $x_{i}$ have degree $d_{i}(1 \leq i \leq n)$. Put $S=\left\{x_{1}, \ldots, x_{m}\right\}$. Put $e=d_{m}-m+1$, then we have to show $\mu_{m} \geq e+1$.

Each point of $S$ has at least $e$ neighbours outside. If each point of $S$ has at least $e+1$ neighbours outside, then we are done by the lemma. And if not, then a point in $S$ with only $e$ neighbours outside is adjacent to all other vertices in $S$, and we are done by the lemma, unless $e=0$.

Suppose first that $\Gamma$ is $K_{m}$ with a pending edge attached, possibly together with some isolated vertices. Then $\Gamma$ has Laplacian spectrum $m+$ $1, m^{m-2}, 1,0^{n-m}$, with exponents denoting multiplicities, and equality holds. And if $\Gamma$ is $K_{m}+K_{2}+(n-m-2) K_{1}$, it has spectrum $m^{m-1}, 2$, $0^{n-m}$, and the inequality holds.

Let $T$ be the set of vertices of $S$ with at most $m-2$ neighbours in $S$. The case $T=\emptyset$ has been treated above. For each vertex $s \in T$ delete all edges except one between $s$ and $X \backslash S$. Now the row of $L_{S}$ indexed by $s$ gets row sum 1 . Since $d_{m}=m-1$ we can always do so. Also delete all edges inside $X \backslash S$, and possible isolated vertices. By interlacing, $\mu_{i}$ has not been increased, so it suffices to show that for the remaining graph $\mu_{m} \geq 1$.

Again consider the partition of $X$ into $m+1$ parts consisting of $\{s\}$ for each $s \in S$, and $X \backslash S$, and let $Q$ be the corresponding quotient matrix of $L$. By interlacing it suffices to show that the second smallest eigenvalue of $Q$ is at least 1 . Put $r=|X \backslash S|$ and $t=|T|$, then $0<r \leq t$, and

$$
Q=\left(\begin{array}{ccc}
m I-J & -J & \mathbf{0} \\
-J & L_{T} & -\mathbf{1} \\
\mathbf{0}^{\top} & -\mathbf{1}^{\top} / r & t / r
\end{array}\right)
$$


( $J$ is the all-ones matrix, and $\mathbf{0}$ and $\mathbf{1}$ denote the all-zeros and the all-ones vector, respectively). Now $Q$ has a $3 \times 3$ quotient matrix

$$
R=\left(\begin{array}{ccc}
t & -t & 0 \\
t-m & m-t+1 & -1 \\
0 & -t / r & t / r
\end{array}\right)
$$

The three eigenvalues of $R$ are $0 \leq x \leq y$ (say). We easily have that

$$
(1-x)(1-y)=\operatorname{det}(I-R)=t-1+(m-1)(t / r-1) \geq 0,
$$

which implies that $x \geq 1$ ( since $x \leq y \leq 1$ contradicts $x+y=\operatorname{trace} R>$ $m+1)$. These three values are also eigenvalues of $Q$ with (right) eigenvectors constant over the partition. The remaining eigenvalues have (left) eigenvectors that are orthogonal to the characteristic vectors of the partition, and these eigenvalues remain unchanged if a multiple of $J$ is added to a block of the partition of $Q$. So they are also eigenvalues of

$$
Q^{\prime}=\left(\begin{array}{ccc}
m I & O & \mathbf{0} \\
O & L_{T} & \mathbf{0} \\
\mathbf{0}^{\top} & \mathbf{0}^{\top} & 1
\end{array}\right),
$$

which are at least 1 since $L_{T}=L(T)+(m-t+1) I$ and $L(T)$ is positive semidefinite. So we can conclude that $\mu_{m} \geq 1$.

\section{$5 \quad$ Equality}

There are many cases of equality (that is, $\mu_{m}=d_{m}-m+2$ ), and we do not have a complete description.

For $m=1$ we have equality, i.e., $\mu_{1}=d_{1}+1$, if and only if $\Gamma$ has a vertex adjacent to all other vertices.

For $m=n$ we have equality, i.e., $0=\mu_{n}=d_{m}-m+2$, if and only if the complement of $\Gamma$ has maximum degree 1.

The path $P_{3}=K_{1,2}$ has Laplace eigenvalues $3,1,0$ and degrees $2,1,1$ with equality for $m=0,1,2$, and is the only graph with equality for all $m$.

The complete graph $K_{m}$ with $a$ pending edges attached at the same vertex has spectrum $a+m, m^{m-2}, 1^{a}$, 0 , with exponents denoting multiplicities. Here $d_{m}=m-1$, with equality for $m$ (and also for $m=1$ ).

The complete graph $K_{m}$ with $a$ pending edges attached at each vertex has spectrum $\frac{1}{2}\left(m+a+1 \pm \sqrt{(m+a+1)^{2}-4 m}\right)^{m-1}, a+1,1^{m(a-1)}, 0$, with $\mu_{m}=a+1=d_{m}-m+2$.

The complete bipartite graph $K_{a, b}$ has spectrum $a+b, a^{b-1}, b^{a-1}, 0$. For $(a=1$ or $a \geq b)$ and $b \geq 2$ we have $d_{2}=a=\mu_{2}$. This means that all graphs $K_{1, b}$, and all graphs between $K_{2, a}$ and $K_{a, a}$ have equality for $m=2$.

The following describes the edge-minimal cases of equality.

Proposition 3 Let $\Gamma$ be a graph satisfying $\mu_{m}=d_{m}-m+2$ for some $m$, and such that for each edge e the graph $\Gamma \backslash$ e has a different $m$-th largest degree or a different $m$-th largest eigenvalue. Then one of the following holds. 
(i) $\Gamma$ is a complete graph $K_{m}$ with a single pending edge.

(ii) $m=2$ and $\Gamma$ is a complete bipartite graph $K_{2, d}$.

(iii) $\Gamma$ is a complete graph $K_{m}$ with a pending edges attached at each vertex. Here $d_{m}=m+a-1$.

Proof This is a direct consequence of the proof of the main result.

Many further examples arise in the following way. Any eigenvector $u$ of $L=L(\Gamma)$ remains eigenvector with the same eigenvalue if one adds an edge between two vertices $x$ and $y$ for which $u_{x}=u_{y}$. If $\Gamma$ had equality, and adding the edge does not change $d_{m}$ or the index of the eigenvalue $\mu_{m}$, then the graph $\Gamma^{\prime}$ obtained by adding the edge has equality again.

The eigenvector for the eigenvalue $a+1$ for $K_{m}$ with $a$ pending edges attached at each vertex, is given by: 1 on the vertices of degree 1 , and $-a$ on the vertices in the $K_{m}$. So, equality will persist when arbitrary edges between the outside vertices are added to this graph, as long as the eigenvalue keeps its index and $d_{m}$ does not change.

The eigenspace of $K_{a, b}$ for the eigenvalue $a$ is given by: values summing to 0 on the $b$-side, and 0 on the $a$-side. Again we can add edges.

For example, the graphs $K_{2, d}$ with $d \geq 2$ have $d_{2}=d=\mu_{2}$ with equality for $m=2$. Adding an edge on the 3 -side of $K_{2,3}$ gives a graph with spectrum $5,4,3,2,0$, and the eigenvalue 3 is no longer 2 nd largest. Adding an edge on the 4 -side of $K_{2,4}$ gives a graph with spectrum 6,4 , $4,2,2,0$, and adding two disjoint edges gives $6,4,4,4,2,0$, and in both cases we still have equality for $m=2$.

\section{References}

[1] R. Grone \& R. Merris, The Laplacian spectrum of a graph II, SIAM J. Discr. Math. 7 (1994) 221-229.

[2] Ji-Ming Guo, On the third largest Laplacian eigenvalue of a graph, Lin. Multilin. Alg. 55 (2007) 93-102.

[3] W. H. Haemers, Interlacing eigenvalues and graphs, Linear Alg. Appl. 226-228 (1995) 593-616.

[4] Jiong-Sheng Li \& Yong-Liang Pan, A note on the second largest eigenvalue of the Laplacian matrix of a graph, Lin. Multilin. Alg. 48 (2000) 117-121. 